\title{
High Availability Acoustic Positioning for Targets Moving in Poor Coverage Areas
}

\author{
Teodoro Aguilera, Joaquín Aparicio, Member IEEE, Fernando J. Álvarez, Senior Member IEEE
}

\begin{abstract}
Currently, most acoustic positioning strategies are based on broadband coding and the pulse compression technique to obtain better accuracy and robustness against in-band noise. However, the accuracy and robustness of these systems is often seriously damaged in areas of low coverage and/or affected by multipath. This work proposes a High Availability Positioning Algorithm (HAPA) that optimally processes the received signal to improve positioning results throughout the whole location area. This algorithm validates the Time-of-Arrival (ToA) from each beacon, corrects their Doppler shift and compensates for multipath when it exists. If the number of validated beacons is enough to obtain a position, the algorithm tries to position with their optimal combination; if not, it tries to position by incorporating additional ToA candidates for the non-validated beacons.

The HAPA performance has been evaluated through a set of experimental tests to compare its results with those obtained by the Robust Positioning Algorithm (RPA) developed by the authors in a previous work, and by a Classical Positioning Algorithm (CPA) based on the emission of chirps. In particular, when using a mobile robot to follow trajectories that cross areas with low coverage and affected by multipath, the HAPA maintains an availability of $97.89 \%$, while the RPA drops to $83.25 \%$ and the CPA to $50.19 \%$.
\end{abstract}

Index Terms-Acoustic Local Positioning System, Efficient Signal Processing, High Availability, Low Coverage, Multipath, Doppler, Ultrasound, Spread-spectrum

\section{INTRODUCTION}

Local positioning is an active research topic that has attracted the attention of the instrumentation and measurement community for more than a decade now. During this period, a variety of systems have been proposed based on different technologies such as light [1], ultra-wideband radar [2], [3], [4], magnetic fields [5], [6], [7], RFID [8], inertial sensors [9] and acoustics [10], [11], [12]. This latter has been one of the most widely developed systems since the appearance of the first works at the end of the former century [13], [14], [15], and today it is considered a reliable solution that provides centimeter accuracy with maximum coverage of tens of meters [16], [17]. During this period of intense research, Acoustic Local Positioning Systems (ALPS) have incorporated broadband signal coding and the pulse compression technique to improve their accuracy and robustness against noise [18], [19], as well as complex algorithms to mitigate detection hindering phenomena such as multiple access interference [20], [21] or multipath propagation [22], [23].

Teodoro Aguilera and Fernando J. Álvarez are with the Department of Electrical Engineering, Electronics and Automation, University of Extremadura, Badajoz,06006, Spain, e-mails: teoaguibe@unex.es, fafranco@unex.es.

Joaquín Aparicio is with the Department of Informatics, University of Oslo, 0373 Oslo, Norway (e-mail: joaqapar@ifi.uio.no).
Today, ALPS developers find motivation in the positioning and tracking of moving targets in real, complex environments. These targets can be either people using their smartphones as intelligent signal receivers that compute their position to provide the user with location-based services [24], [25], or flying drones with applications in warehouse logistics [26], [27], [28]. This ambitious goal implies new design challenges.

The first of these challenges is the Doppler effect associated with the movement of the target. The consequences that this phenomenon can have on the detection of broadband acoustic signals have been already analyzed in some works [29], [30]. In [31], two of the authors of this work proposed a novel Doppler compensation algorithm that computed the time shift undergone by the detection pulse to estimate the position of a moving target accurately. A second challenge that has not attracted much attention from acoustic LPS researchers is the operation in poor coverage areas, which arise in complex environments with real-world obstacles. Most ALPS receivers avoid false detections by using an energy threshold below which no correlation peak is validated. Unfortunately, this threshold may be above actual arrival peaks in poor coverage areas that will not be detected, giving rise to an underdetermined system of positioning equations with no single solution. This situation is typically tackled by rendering the last valid position or an estimated evolution of this last valid position. Other receivers make no use of an energy threshold and always assume a detection by searching for the highest amplitude peak in an evaluation window. Under poor coverage, this approach may validate false arrivals that will eventually generate an erroneous estimation of the position or even cause the positioning algorithm to diverge. Statistical analysis can be used to discard these outliers when enough measurements are taken in similar conditions. However, none of these solutions is useful when the need is to track fast-moving targets.

Recently, the authors have proposed a solution to this problem that increases the availability of chirp-based Classical Positioning Algorithms (CPA) between 20 and 25\% when tracking a mobile robot with speeds up to $2 \mathrm{~m} / \mathrm{s}$ in areas with limited coverage [32]. This solution consists of a complex signal encoding scheme based on the use of an OFDM modulated Complementary Set of Sequences, which is attached to the Doppler tolerant combination of a chirp and a carrier pulse previously described in [31]. This chirp + carrier header was used to baseband demodulate the appended tail and thus validate the detection of weak signals. However, despite the notably enhanced performance of the new system, there was still a wide room for improvement, which has been the main motivation for the present work. 
Here, we present an evolved version of the Robust Positioning Algorithm (RPA) proposed in [32], where we have worked on the limitations that we found in the experimental tests. This new High Availability Positioning Algorithm (HAPA) raises the system performance close to its maximum achievable value. There are several differences between both algorithms that make this performance improvement possible, and are worth noticing:

- Regarding the emitted signal, RPA used only two orthogonal codes in the validation step: one for the reference beacon, and another one for the rest. HAPA uses five orthogonal codes, one for each beacon. Additionally, the order of the chirp and pulse has been exchanged to reduce the impact of multipath on the Doppler shift estimation.

- If RPA loses the reference beacon, positioning is not possible. Thanks to the new design, HAPA is always able to choose a reference beacon from the available arrivals in each frame.

- HAPA incorporates a more efficient multipath detection algorithm than RPA, based on a log of valid ToA from previous calculated positions. It is combined with a multipath compensation algorithm, that even allows to recover peaks missed by the chirp peak detector. The combination of the two algorithms makes HAPA more robust to multipath than RPA.

- With respect to availability, RPA only tries to position with existing validated peaks. In addition, HAPA also proposes possible candidate peaks if there are not enough validated peaks, and tries all possible beacon combinations to get a valid position.

The rest of the paper is organized as follows. Section II describes the emitter and receiver modules, as well as the signal coding scheme of the proposed system. In Section III the new HAPA is proposed. Section IV presents and discusses a set of experimental results obtained in a real environment with a mobile robot. Finally, Section V highlights the most relevant conclusions that can be drawn from this work.

\section{Positioning System Design}

The system used is composed of an emitter and receiver modules operating in a laboratory environment on a positioning surface of $2.4 \times 2.4 \mathrm{~m}^{2}$. Each of the system components and the designed acoustic signals are described below.

\section{A. Emission Module}

The module for the signal emission has been developed on a Printed Circuit Board (PCB), which includes an NXP LPC1768 microcontroller [33], a Digital-to-Analog Converter (DAC) with 8-bit resolution, a stage for signal conditioning and an AC/DC converter to connect the board to the mains.

Five Prowave 328ST/R160 ultrasonic transducers [34] have been connected to this electronic module. According to their characteristics, the optimum working range for these transducers has been established between 36 and $44 \mathrm{kHz}$, since it is in this bandwidth where they offer the best flat response. Moreover, the location of each transducer has been established with millimeter precision using a laser rangefinder [35].

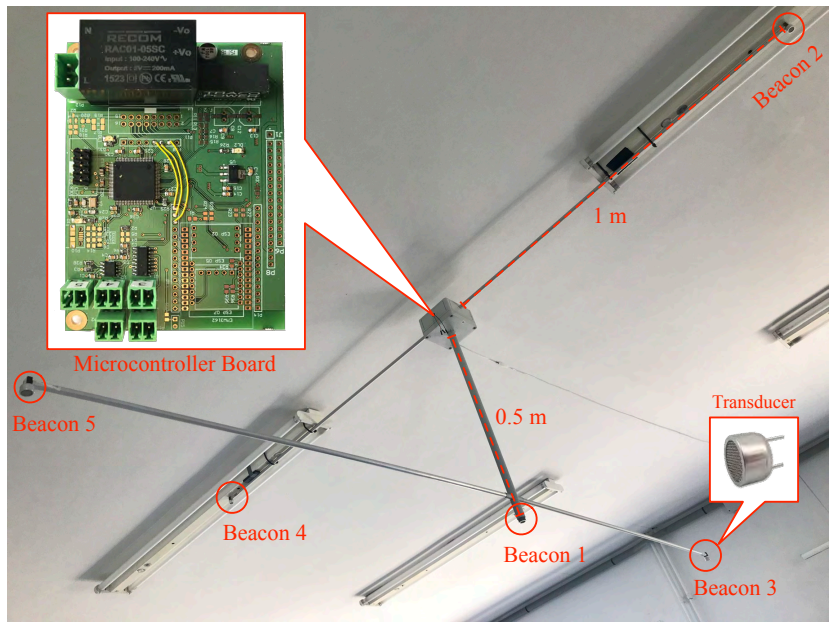

Fig. 1: Emitter module description.

Fig. 1 shows the complete emitter module located on the laboratory ceiling. Each of its components has been labeled, and a view of its interior is provided showing the PCB.

\section{B. Signal Design}

The design of the transmitted signals, which is an evolved version of previous work from the authors [32], is now described.

The transmitted signals consist of three components: 1) a $10 \mathrm{~ms}$ carrier with a frequency of $40 \mathrm{kHz}, 2$ ) a $10 \mathrm{~ms}$ chirp transmitted between 36 and $44 \mathrm{kHz}$, and 3) a binary code modulated in OFDM. The carrier allows compensating for the Doppler effect experienced by the signal, whereas the chirp provides candidate arrivals. Beacon identification is obtained from the last signal component: it consists of a unique code for each beacon, which validates or discards a candidate arrival and identifies the transmitter. This validation process will be explained in more detail in Section III.

In order to choose a coding family, we evaluated the performance in a preliminary study of the best set of five orthogonal codes from two different families: Kasami codes [36], and Complementary Set of Sequences (CSS) [37], both with similar lengths (63 and 64 bits, respectively). Performance was based on the bound, defined as the ratio of the highest-amplitude sidelobe to the main auto-correlation peak, where a value closer to 0 means better orthogonality. These values were 0.222 for the Kasami set and 0.375 for the CSS, so Kasami codes were selected for this work.

The OFDM technique has been used to modulate the Kasami codes due to its simple modulation and demodulation process and its easy channel equalization in the frequency domain. In addition, OFDM also offers some immunity to impulsive noise and even certain robustness to multipath when using cyclic prefixes [38]. The 63 bits composing each Kasami code have been mapped in Quadrature Phase-Shift Keying (QPSK) and assigned to 32 subcarriers inside a bandwidth of $8 \mathrm{kHz}$. This modulation generates signals of $4 \mathrm{~ms}$ duration. Moreover, a same-duration cyclic prefix has been included, resulting in a total modulated code duration of $8 \mathrm{~ms}$. Channel 


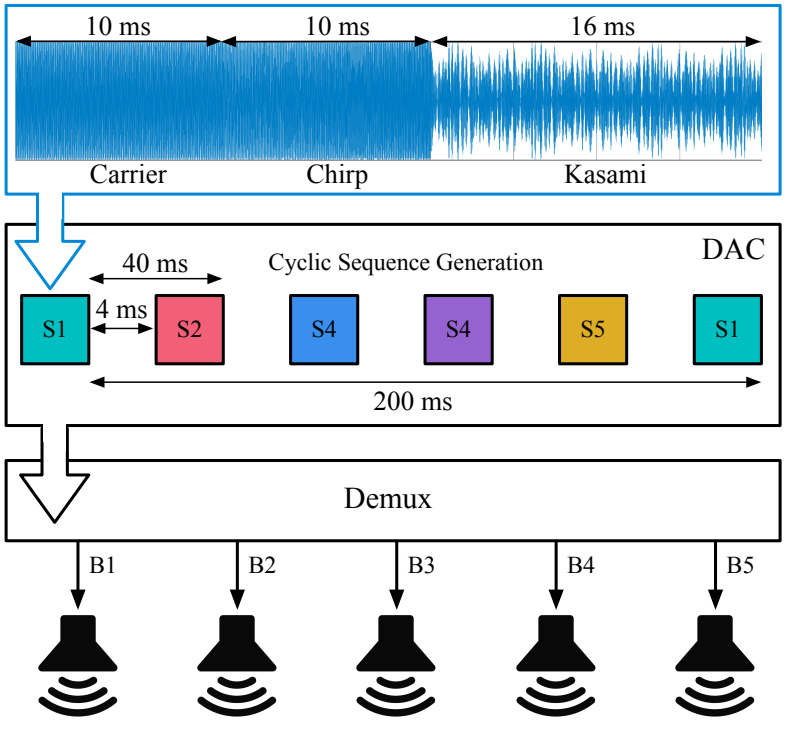

Fig. 2: Signal composition and emission architecture.

equalization and demodulation at the receiver will be carried out with the help of 32 OFDM-modulated pilot symbols that have been transmitted on the same subcarriers, also including a cyclic prefix. Pilot symbols add an extra $8 \mathrm{~ms}$ to the signal duration. Therefore, the third signal component total duration is $16 \mathrm{~ms}$, and the transmitted signal total duration, including the carrier and the chirp, is $36 \mathrm{~ms}$.

Fig. 2 shows an example of the proposed signal design, which illustrates the three components of the transmitted signals and the transmission schedule of the five beacons. This schedule is based on a T-CDMA protocol (Time Code Division Multiple Access), where each transmission is encoded and transmitted in different slots since the board only has one DAC. The slot duration is $40 \mathrm{~ms}$.

It is worth noticing that, in order to compare the performance of the proposed HAPA with an approach based solely on chirps (CPA), we used two different chirps in the signal design: one is created with a linear increase of frequencies (upchirp), and it is transmitted by the "reference beacon" in the CPA (Beacon 1, B1); the other is designed with a linear decrease of frequencies (downchirp), and it is transmitted by the other four beacons (B2 to B5). We remark that in the HAPA, no beacon identification is based on the chirps. We only keep the arrival times and amplitudes for the list of candidate arrivals.

\section{Reception Module}

A wide-bandwidth ultrasonic microphone [39] has been used as a receiving device. This microphone is connected to a signal acquisition system with a sampling frequency $f_{s}=500 \mathrm{kHz}$. This sampled signal $y(t)$ is stored in a laptop for further processing by the HAPA. The stored data are divided into $240 \mathrm{~ms}$ frames with an overlap of $40 \mathrm{~ms}$ between them. Since the five beacons emission time takes $200 \mathrm{~ms}$, this procedure ensures that all beacons will always be received in each frame, and one beacon is received twice. In this way, there will always be information from all beacons to allow 2D positioning with an update rate of $5 \mathrm{~Hz}$.

\section{High Availability Positioning Algorithm}

This section explains all the steps followed by the proposed HAPA, shown in Fig. 4. The algorithm loads a $240 \mathrm{~ms}$ frame. A bandpass filter is firstly applied to keep the signal corresponding to the transducer bandwidth, and to reduce the noise. The filter output is upsampled then to $1 \mathrm{MHz}$, in order to increase the resolution in the ensuing correlation with the chirps.

A matched filter is applied to the upsampled signal to detect the upchirp, and another matched filter looks for the downchirps. In each of these correlation signals, we used a peak detector [40] to look for the chirp arrivals by considering a sliding window of $40 \mathrm{~ms}$ with a $10 \mathrm{~ms}$ overlap, so no chirp is missed. We set the thresholds of the peak detector to allow low-amplitude peaks to be detected, in order to compensate for the near-far effect. As a side effect, some spurious peaks caused by multipath and correlation noise will be detected too. When all the windows in the frame are processed, a list of $n_{c}$ candidates containing their arrival times is obtained.

Subsequently, a peak validation process is carried out ${ }^{(1)}$. The first step is to check the Doppler shift experienced by the received signal. This is done on the attached carrier, by calculating the Fast Fourier Transform (FFT) and comparing the frequency at the spectrum maximum with the transmitted carrier frequency. In addition to Doppler shift estimation, this steps also allows to remove some of the spurious peaks detected before. If the estimated Doppler shift of a particular arrival is greater than $1 \mathrm{kHz}$, it is concluded that the corresponding peak does not come from a valid transmission (it is assumed that the expected carrier was not found), and it is discarded. Therefore, for a carrier of $40 \mathrm{kHz}$, this $1-\mathrm{kHz}-$ threshold rejects speeds higher than $8.5 \mathrm{~m} / \mathrm{s}$, approximately. This speed is large enough for indoor environments. After this step, the list of candidate arrivals is reduced to $n_{c}^{\prime}$.

The validation step based on the Kasami code follows next for each of the remaining $n_{c}^{\prime}$ candidate arrivals. The algorithm extracts the samples corresponding to the OFDM signals from the pilot symbols and the Kasami code, they are downsampled to $f_{s}=500 \mathrm{kHz}$, and a resampling operation compensates the experienced Doppler shift, as calculated on the previous step. The pilot symbols are demodulated next. The received pilots $Y_{p}[k]$ are contained in $K$ subcarriers:

$$
Y_{p}[k]=H[k] \cdot X_{p}[k],
$$

where $k=1, \ldots, K$. The channel response $H[k]$ is obtained from (1), since the transmitted pilots $X_{p}[k]$ are known:

$$
H[k]=Y_{p}[k] / X_{p}[k]
$$

Analogously, the Kasami code is demodulated next obtaining $Y[k]$. The equalized symbols $X^{e q}[k]$ can be calculated then from (3):

$$
X^{e q}[k]=\frac{Y[k]}{H[k]}
$$

This step presupposes a constant channel response during $16 \mathrm{~ms}$, that is, the duration of the modulated pilots and Kasami code, which is a reasonable assumption.

\footnotetext{
${ }^{1}$ Superscript indicates a particular block in Figure 4.
} 
The equalized symbols are then demapped, and bit decision provides the binary samples $S[i]$ following (4):

$$
S[i]=\left\{\begin{array}{r}
1, \text { if } X^{e q}[i]>0 \\
-1, \text { if } X^{e q}[i]<0
\end{array}\right.
$$

Once the 63 bits are recovered, correlation operations are conducted against the transmitted codes in $Q$ branches, one for each beacon. Performing the correlation in baseband decreases modulation effects and simplifies the detector since the peak must appear at the sample $L$, where $L$ is the length of the code. The normalized peak amplitudes $\phi^{q}$ at sample $L$ are calculated next at each branch $q$, following:

$$
\phi^{q}=\frac{A^{q}}{L}
$$

where $A^{q}$ is the peak amplitude at sample $L$ and branch $q$.

The detector then looks for the branch with maximum value $\phi=\max \left(\phi^{q}\right)$, for $q=1, \ldots, Q$, which provides beacon identification. Moreover, if $\phi \geq 0.5$, the peak is validated; otherwise, it is marked as a candidate to be further evaluated. After all candidate arrivals have been evaluated, the algorithm keeps only one peak per beacon. In the event that more than one peak is validated for a particular beacon (for example, due to multipath), only the one arriving first is kept. If there is one validated peak and one or more candidates for that beacon, only the validated one is retained. Furthermore, if there are only candidates, the one arriving first is kept. After this step is completed, there are potentially $n$ validated peaks, and $m$ candidate peaks, where $\max (n)=Q$, and $\max (m)=Q-n$. In the system proposed in this work, since we have five beacons, $Q=5$.

Fig. 3 shows an example of this validation step. In this case, three arrivals have been validated $\left(\phi^{q}>0.5\right.$, represented with a circle); two have been marked as candidate for further evaluation $\left(\phi^{q}<0.5\right.$, represented with a square); and two arrivals discovered by the peak detector did not meet the validation criteria (correlation peak not at sample $L$ ), and will not be further processed by the HAPA algorithm.

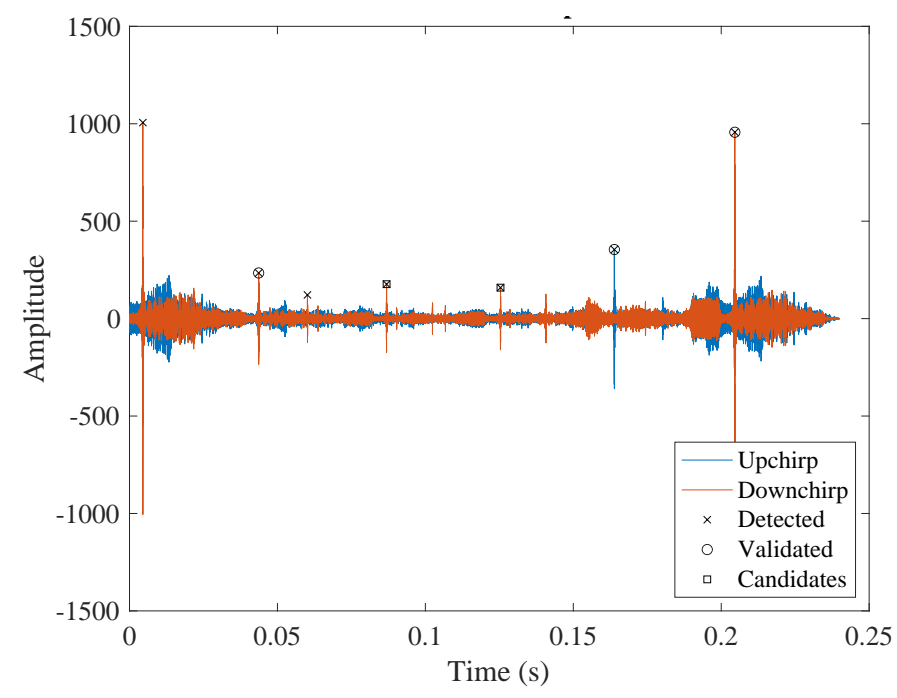

Fig. 3: Detected, validated and candidates correlation peaks.
Potential multipath detections are evaluated next. The algorithm keeps a log of the beacons ToA when a position is successfully obtained using all beacons. If the log is not empty, the new ToA is compared with the mean of the recorded ToA for each beacon. If it deviates more than a certain threshold, it is marked as potential multipath, to be evaluated later. This threshold depends on the environment (walls, beacons locations, ceiling height), so it must be manually calculated. In this case, it was set to $10 \mathrm{~ms}$, which corresponded to multipath coming from floor-ceiling rebounds. A second threshold is set at $40 \mathrm{~ms}$ to remove peaks that would be incorrectly identified at another slot. Once the validation process and multipath detection are completed, the Signal to Noise Ratio (SNR) of each validated peak is calculated.

If all five beacons have a validated peak, their associated Times of Arrival (ToA) are included in a Rotation Beacon Algorithm $^{(2)}$ (RBA). This algorithm searches for the optimal combination of beacons that leads to successful positioning. The algorithm starts by testing with the maximum number of beacons available, five, in this case, making combinations of them in decreasing number until it succeeds in positioning or falls below three beacons required for $2 \mathrm{D}$ positioning. The RBA is used in any case, even when having enough validated beacons, because some of these ToA may be a close multipath and make the positioning algorithm diverge.

For each combination of beacons, the reference beacon ${ }^{(3)}$ is chosen. Since they can be validated or not and can also be potentially affected by multipath, a qualifying factor is assigned. From top to lowest priority, the algorithm will use:

1) A validated beacon with no multipath, with maximum chirp amplitude in the frame.

2) A validated beacon with no multipath, with better chirp amplitude from the ones available.

3) A candidate beacon with no multipath, with maximum chirp amplitude in the frame.

4) A candidate beacon with better chirp amplitude.

Subsequently, the TDMA scheme is unfolded, and the possible Range Doppler Coupling ${ }^{(4)}$ (RDC) associated with each ToA is compensated. The RDC algorithm [31] compensates for the time shift suffered by the chirp correlation peaks due to the Doppler effect. Once these time shifts have been corrected, the Time Difference of Arrivals (TDoA) are calculated according to the chosen reference beacon. Afterwards, the positioning algorithm based on multilateration with Gauss-Newton quadratic error minimisation is applied. If the positioning algorithm converges, i.e. provides a correct position, that position is associated with the receiver. If all the beacons were used in the position estimation, their ToA are stored in the log to check for multipath at later frames; otherwise, the RBA proposes a new combination of beacons, and the positioning process is repeated.

In case of not all beacons being validated after the peak validation $\operatorname{step}^{(1)}$, candidate peaks are selected for the remaining beacons ${ }^{(5)}$. In order to incorporate candidate beacons, the algorithm needs at least two validated beacons, so it can try to position with at least three of them. So, if the number of beacons with validated peaks is not greater than one, the validated peak (if any) and candidate peaks are 


\section{content may change prior to final publication. Citation information: DOI 10.1109/TIM.2021.3106684}

IEEE TRANS. ON INTRUMENTATION AND MEASUREMENT, VOL. XX, NO. XX, XXXXXX 2021

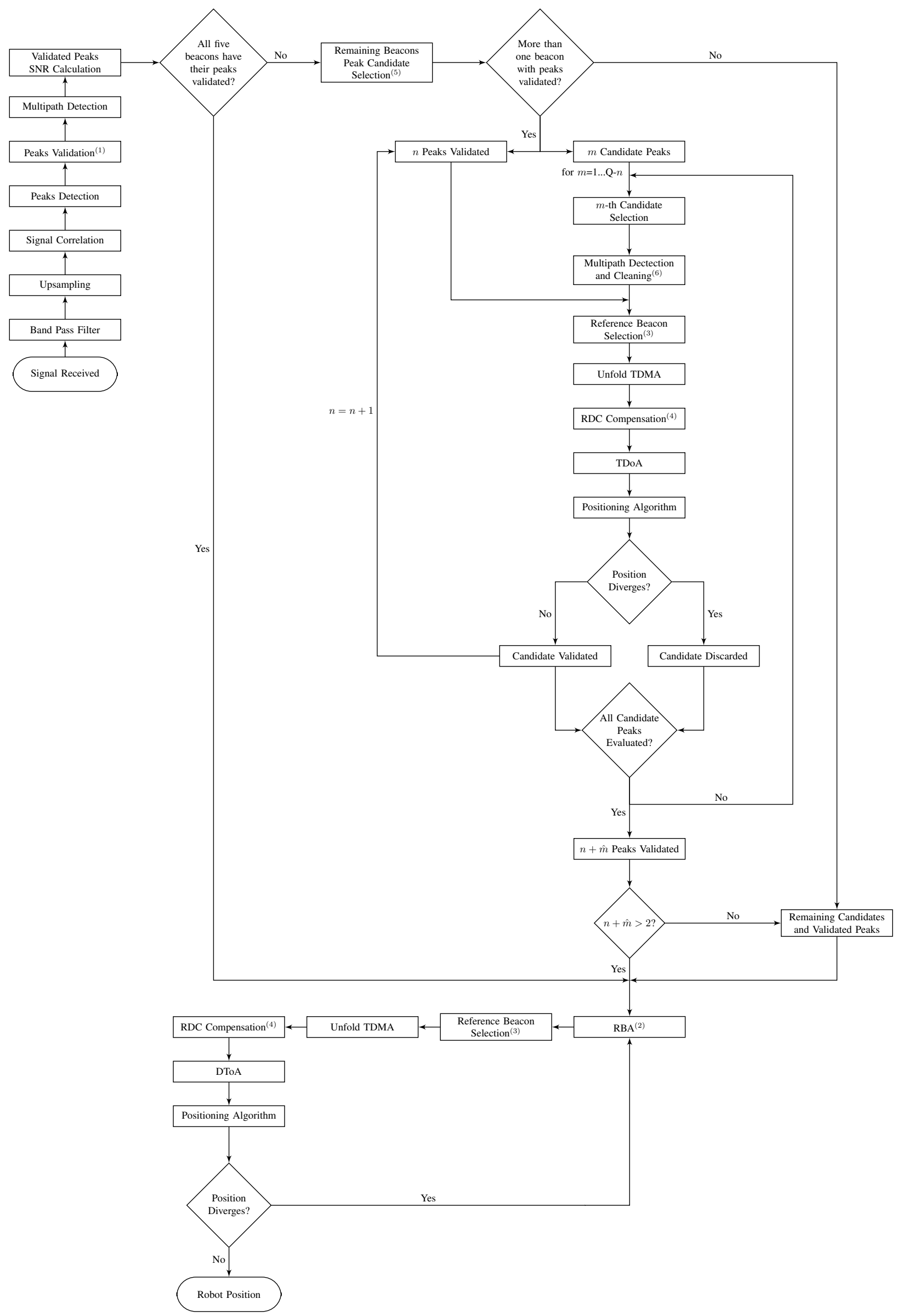

Fig. 4: High availability positioning algorithm flowchart. 
passed directly to the RBA. On the contrary, if the number of validated peaks is greater than one, the remaining $Q-n$ beacons' candidate peaks are evaluated. These candidate peaks are processed one at a time. First, the algorithm evaluates whether it is affected by multipath by checking the ToA log. If it is, its associated Line of Sight Time of Flight (LoS-ToA) is searched. For this purpose, the Multipath Compensation Algorithm (MCA) [23] adapted to the signal coding used in this work has been used. The MCA is based on the accurate impulse response estimation from which the LoS-ToA for each channel is obtained. The algorithm takes as ToA-LoS the first channel's impulsive response peak in chronological order that exceeds a certain threshold. This threshold is heuristically set as a percentage of the highest amplitude impulsive response peak.

Next, the reference beacon is selected from the set of beacons consisting of those with validated peaks and the candidate's beacon under evaluation, following the same criteria as before. The ToA of this set of beacons, with at least three components, are used to determine whether it is possible to obtain a correct position. For this, the TDMA is unfolded, the RDC of each ToA is compensated, and the TDoA are obtained. If the positioning algorithm converges, the candidate peak is validated and added to the previously $n$ validated ones. If the algorithm diverges, the candidate is discarded. Subsequently, the same procedure is repeated for each of the remaining peak candidates. This step allows to validate an extra $\hat{m}$ candidates, where $\hat{m}$ is between 0 and $m$.

Finally, if the number of validated peaks $(n+\hat{m})$ is greater than two, their associated beacons are passed directly to the RBA to obtain the positioning as explained above. Conversely, if $n+\hat{m} \leq 2$, the HAPA uses the set of beacons associated with validated and candidate peaks to supply the RBA. Therefore, the HAPA prioritises validated peaks when computing a position. Only when the number of validated peaks is not enough, i.e. less than three, the algorithm tries to get a position with a combination of validated and candidate peaks.

\section{EXPERIMENTAL SySTEM EVALUATION}

The results obtained in the system experimental evaluation are presented below. Firstly, a system characterisation has been conducted to evaluate its properties on a grid of points over the whole positioning area. Secondly, several trajectories with the robot in motion have been carried out to evaluate the system's performance.

\section{A. System Characterization}

First, a grid of 49 points with a $0.4 \mathrm{~m}$ separation was designed to conduct the system characterization. At each grid point, one hundred measurements have been taken, placing the microphone at the height of $0.755 \mathrm{~m}$.

The Signal to Noise Ratio (SNR) of each beacon has been measured at all grid points. Fig. 5 shows each beacon's isometric levels for SNR values equal to or less than $2 \mathrm{~dB}$, thus representing the regions of lower coverage. In particular, it can be seen how Beacon 3 (B3), in purple, has a low coverage area in the left part of the positioning area, or how Beacon 2
(B2), in orange, has also low intensity in the upper left area. In general, we can see how the left part of the positioning area is the one with the most significant coverage problems, while the area delimited by the position of $\mathrm{B} 1, \mathrm{~B} 2$ and $\mathrm{B} 3$ is the region with the best coverage. Overall, for all 5 beacons and 49 test points, a minimum SNR value of $-2.45 \mathrm{~dB}$ and a maximum value of $22.83 \mathrm{~dB}$ were measured during the tests.

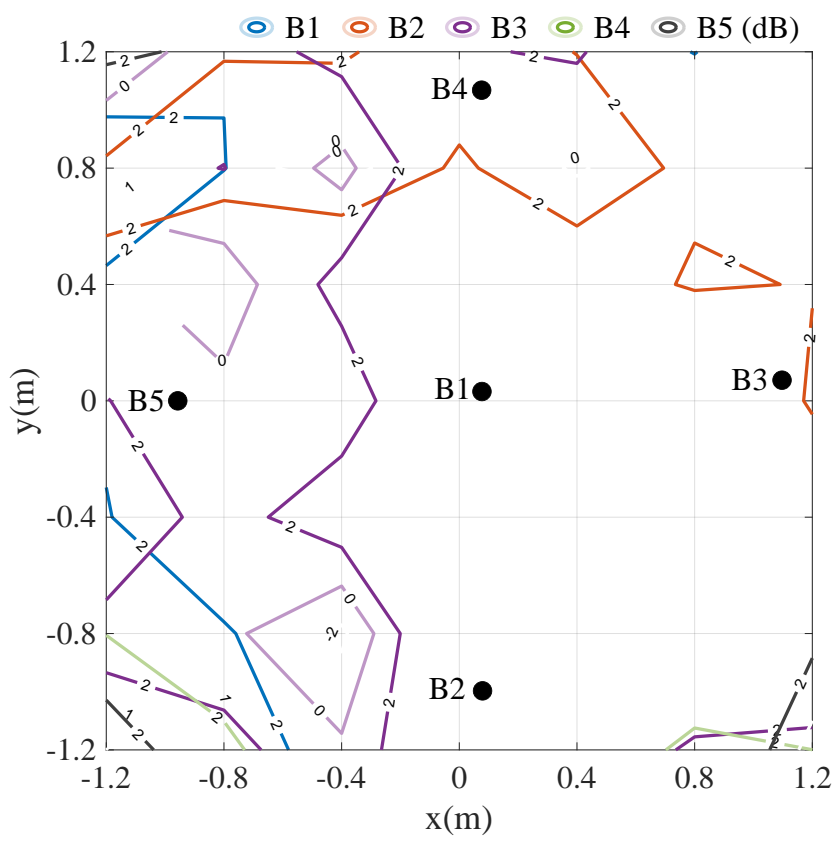

Fig. 5: Isometric SNR levels in (dB).

The system's availability is evaluated next, i.e. the percentage of successful positions obtained in the set of one hundred measurements taken at each one of the grid points. The results obtained in these experiments have been compared using three algorithms: 1) the Classical Positioning Algorithm (CPA) without ToA validation, 2) the Robust Positioning Algorithm (RPA) developed in [32], and 3) the High Availability Positioning Algorithm (HAPA) proposed in this work.

Figure 6a shows the results for the CPA. It can be noticed that there are a large number of positions in the grid where the system has very low or even zero availability. These poor results are mainly in those areas with low coverage illustrated in Fig. 5. Only those test points with an SNR higher than $2 \mathrm{~dB}$ and where the received signals are not affected by multipath or Doppler effect offer acceptable availability values.

Figure $6 c$ represents the results obtained when the signal received at each test point is processed with the RPA. This algorithm performs a more elaborate signal processing, where the candidate ToA are subjected to a process of validation and Doppler compensation. This algorithm achieves significantly better results than those obtained with the CPA. In particular, it can be seen that in low coverage areas, the number of points with low or no availability has been reduced. However, it can also be observed that in the peripheral zones, there are still specific test points where the availability is low, mainly due to strong multipath or loss of the reference beacon. 


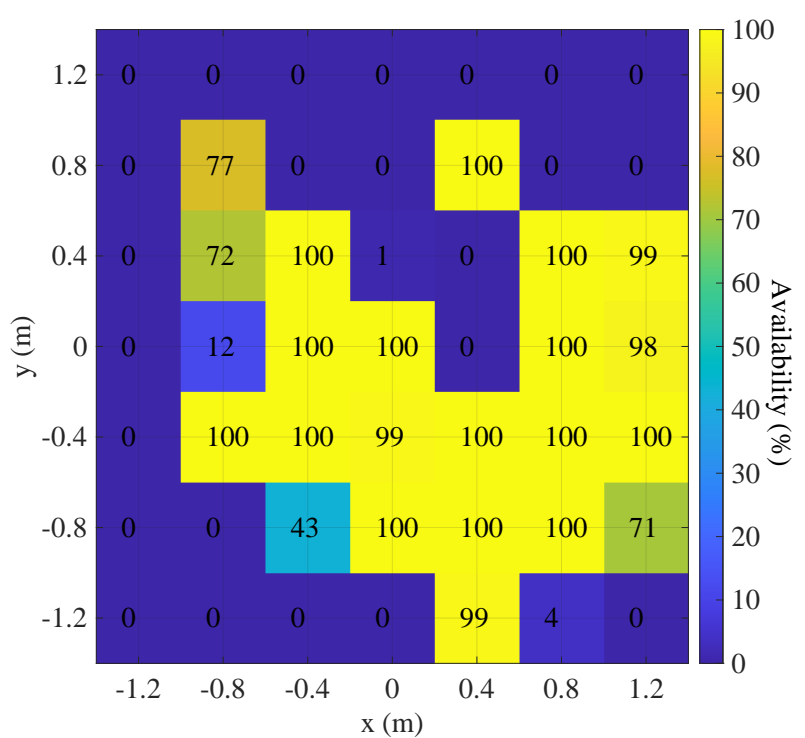

(a) Availability for CPA.

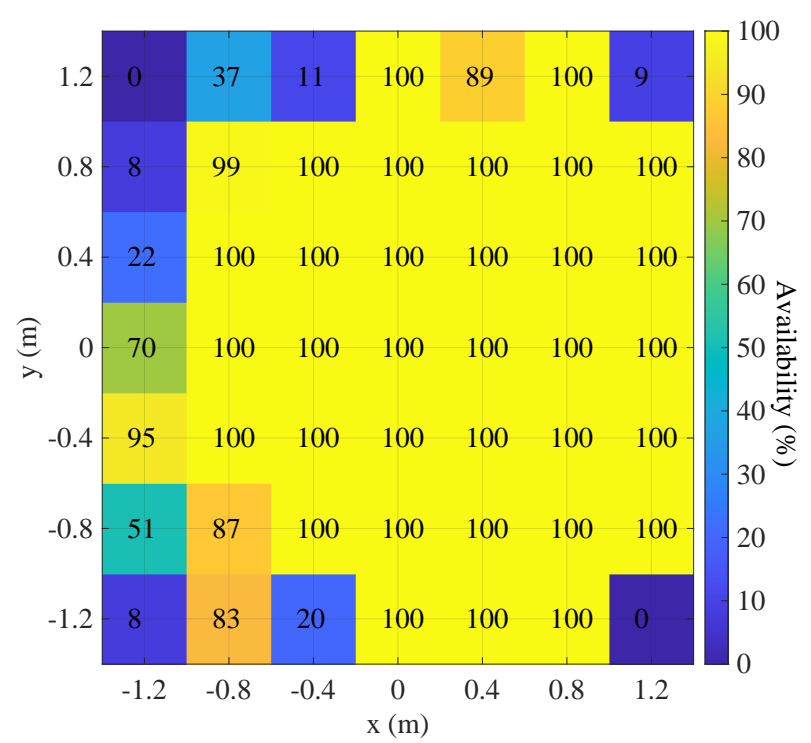

(c) Availability for RPA.

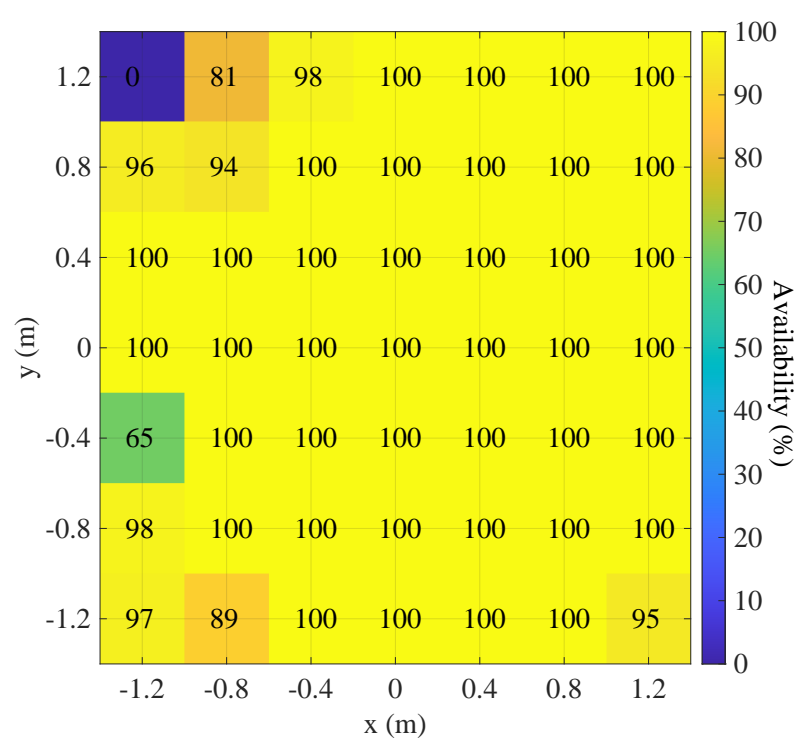

(e) Availability for HAPA.

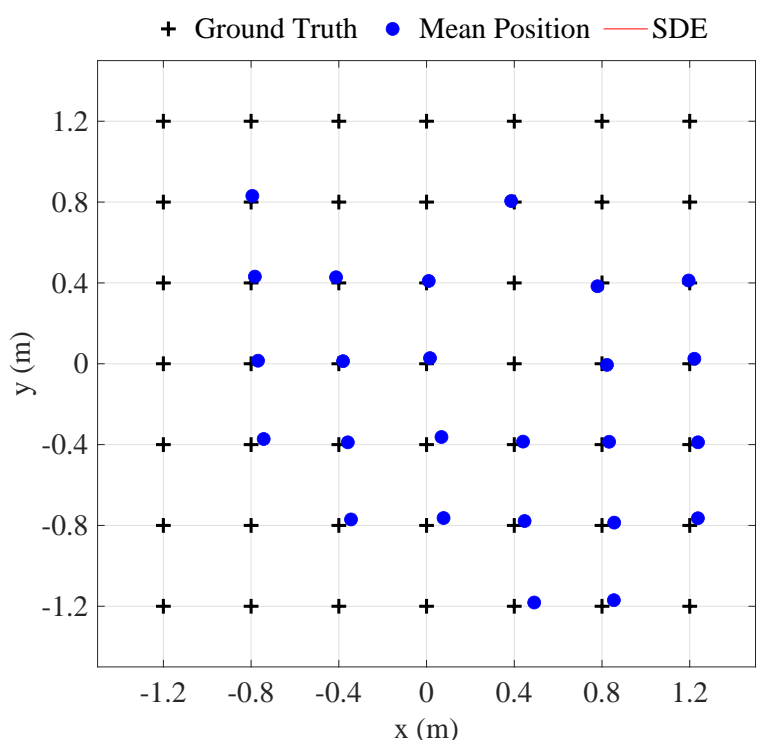

(b) Mean error and SDE for CPA.

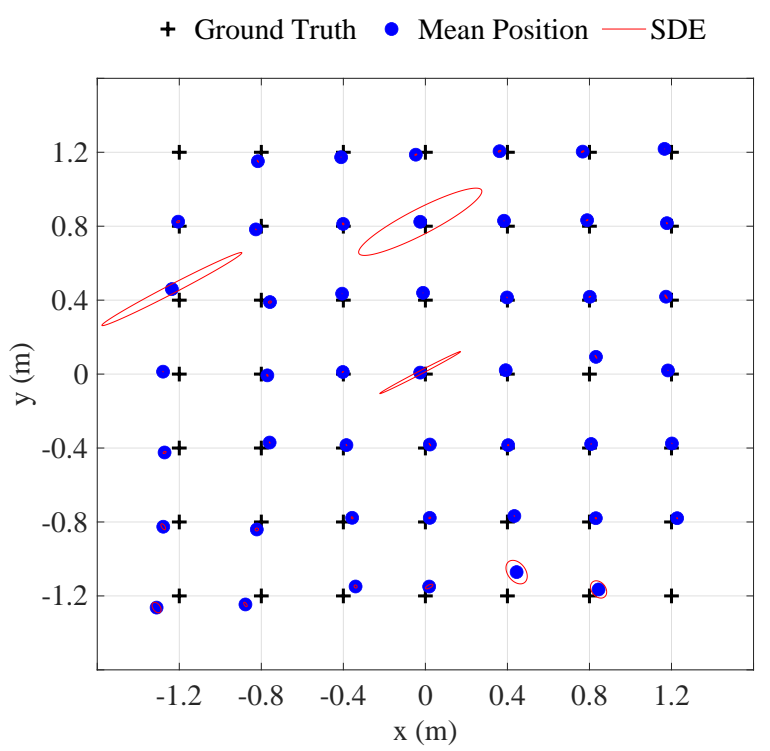

(d) Mean error and SDE for RPA.

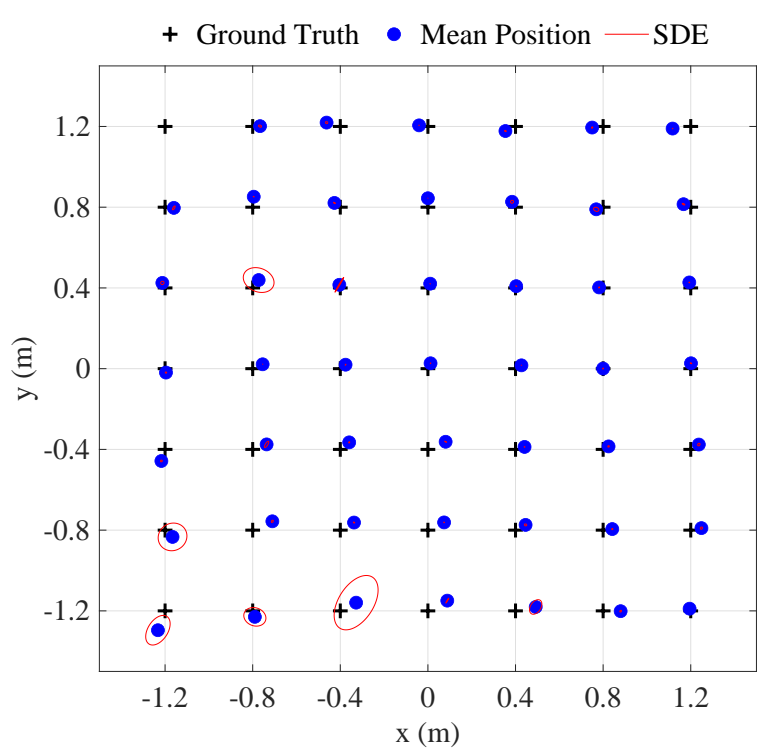

(f) Mean error and SDE for HAPA.

Fig. 6: System availability and accuracy provided by each algorithm. 
Finally, Fig. 6e shows the results obtained with the HAPA. In this case, the results are even better than those obtained with the RPA. It can be seen that almost all the test points have an availability close to or equal to $100 \%$. There are only two test points where the availability is low. The first one is the point $(-1.2,-0.4)$ with a value of $65 \%$. This low availability was caused by the incorrect detection of $\mathrm{B} 1$ on the rest $35 \%$ of measurements, causing the Gauss-Newton algorithm to diverge since only three beacons were available, including B1. The second one is $(-1.2,1.2)$ with $0 \%$ of availability. This test point is in a low coverage area and close to a corner, strongly affected by multipath. The signals received there are so weak and deteriorated by multipath that the algorithm is not capable of retrieving any position.

Additionally, the Mean Error (ME) and the 1-sigma Standard Deviation Ellipse (SDE) have been also calculated from each algorithm's positioning results at each test point. The results obtained for the CPA algorithm are presented in Figure $6 \mathrm{~b}$. For this algorithm, successful positioning is obtained only in 25 of the 49 test points. The minimum average error obtained for the set of points is $0.013 \mathrm{~m}$, and the maximum is $0.093 \mathrm{~m}$. Because this algorithm only achieves successful positioning at points with good coverage and free of multipath, the positions obtained are very consistent, and there are practically no deviations around the mean value.

Figure $6 \mathrm{~d}$ shows the results obtained with the RPA. The positioning has been successful in all but two test points, obtaining a minimum mean error of $0.012 \mathrm{~m}$ and a maximum mean error of $0.136 \mathrm{~m}$. This larger maximum mean error, compared to the CPA, is due to the higher availability of the system and the inclusion of problematic grid points affected by multipath and low SNR, as indicated too by the SDE. However, this error can be tolerated in a practical application.

Lastly, Figure of depicts the results related to the HAPA. With this algorithm, positioning is achieved in 48 of the 49 test points and it offers the best positioning results. A minimum mean error of $0.002 \mathrm{~m}$ and a maximum mean error of $0.089 \mathrm{~m}$ are obtained. It can be seen that in the lower left zone of the positioning area, there are some test points with a slight dispersion in the measurements that did not appear for CPA and RPA. Again, this result is justified by the performance improvement introduced by HAPA in areas of poor signal quality. Therefore, since there is now greater availability in these areas and consequently a more significant number of measurements, it is logical that their dispersion increases slightly. The effects of the RBA and multipath correction can be better appreciated now, as the SDE exhibit less area than in the RPA.

\section{B. System Performance for a Moving Target}

In this section, a series of experimental tests have been conducted to determine the system's behaviour under different trajectories. For this purpose, a Pioneer 3DX mobile robot has been equipped with a laptop computer, a signal acquisition system [41], [42] and an ultrasonic microphone [39], as shown in Figure 7a. The mobile robot carries inside a Raspberry Pi connected to its serial port, as depicted in Fig. 7b. This device runs the Raspbian operating system [43] and uses the Advanced Robotics Interface for Applications (ARIA) library [44] through which the robot commands can be executed in Python. These commands are programmed in a script on the laptop and then downloaded and executed in the Raspberry Pi through a Secure SHell (SSH) communication protocol.

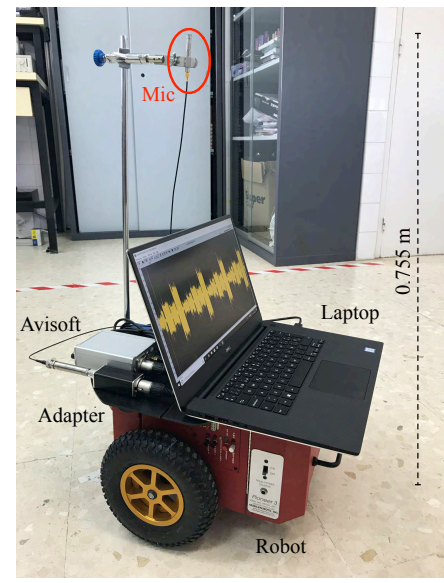

(a) Experimental equipment.

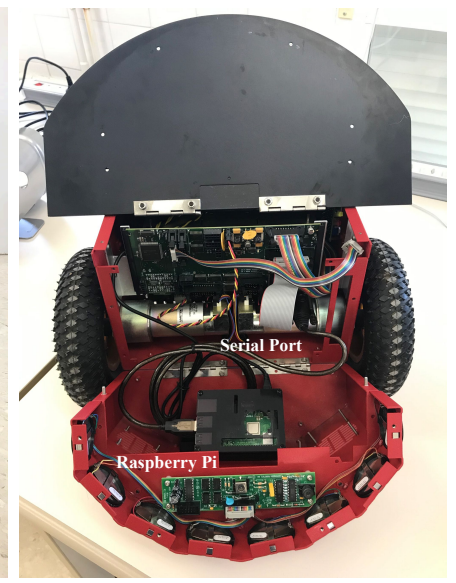

(b) Mobile robot configuration.
Fig. 7: Mobile robot and experimental setup.

During these tests, we decided to remove the resting points at the beginning and end of each trajectory. The elimination of these points establishes a fairer criterion than in [32], given that they have been taken in the centre of the positioning area where the system has good coverage, and under static conditions. Keeping those points would increase the percentage of successful positioning.

In the first test, three square trajectories of 1, 1.5 and 2meter-side are followed, with the robot starting from the center of the square. These three trajectories aim to evaluate the robot's positioning over the positioning area and compare the results obtained with the three algorithms. The results of these tests can be observed for the CPA and HAPA in Fig. 8a. A minimum SNR of $-4.90 \mathrm{~dB}$ and a maximum SNR of $19.70 \mathrm{~dB}$ have been measured in the 1-meter-side trajectory. In this path, the CPA achieved successful positioning in $89.52 \%$ of cases. However, the HAPA increased this percentage to $100 \%$ for the same trajectory. For the 1.5-meter-side trajectory, a minimum SNR of $-6.64 \mathrm{~dB}$ and a maximum SNR of $22.48 \mathrm{~dB}$ were measured. In this trajectory, the CPA was only successful in $83.08 \%$ of cases, while the HAPA succeeded in positioning $100 \%$ of cases. Finally, for the 2-meter-side path, the minimum SNR measured was $-7.93 \mathrm{~dB}$, and the maximum SNR was $25.12 \mathrm{~dB}$. In this trajectory, we observed the largest difference between the maximum and minimum SNR because sometimes the robot is far away from some beacons, and other times it can just pass below one of them, as shown in Figure 8a with yellow circles. Besides, the robot passes close to walls, corners, and objects that accentuate multipath effect during this path. Under these circumstances, the CPA obtained a correct positioning in $50.19 \%$ of cases, while the HAPA achieved $97.89 \%$. 


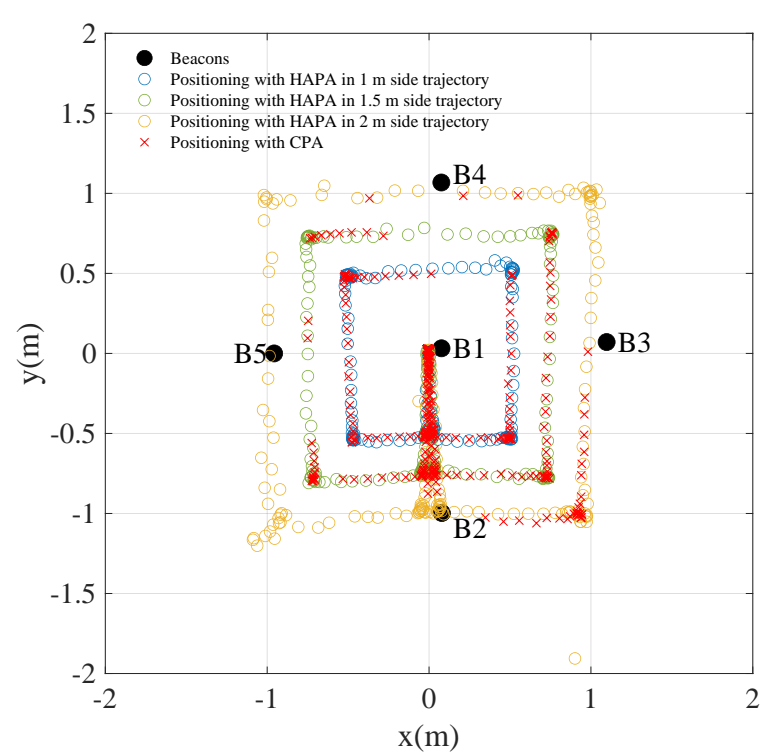

(a) Performance comparison in different square trajectories.

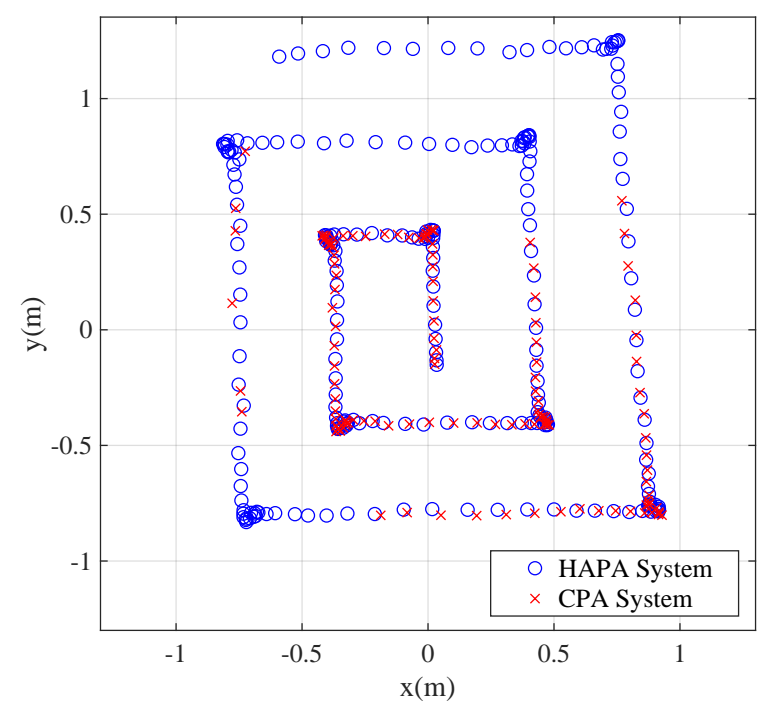

(b) HAPA evolution without position overlapping.

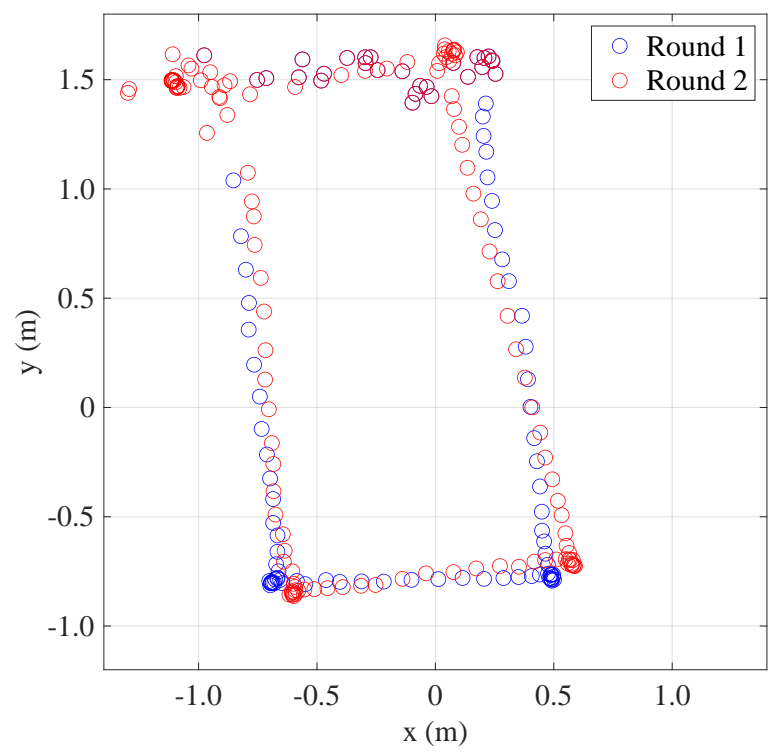

(c) HAPA evolution with position overlapping.

Fig. 8: HAPA performance with different trajectories.
With respect to the results obtained by the RPA, the signals received in [32] have been reprocessed without considering the resting points. In the square trajectory of one meter-side, both algorithms offer $100 \%$ success in positioning. However, in the 1.5-meter-side square trajectory, there is already a slight performance difference. Here, the RPA achieves positioning in $97.64 \%$ of cases, while the HAPA still maintains a $100 \%$. This difference in performance becomes even more pronounced in the 2-meter-side square trajectory. The RPA achieves successful positioning in $83.25 \%$ of cases while, as described above, the HAPA obtains $97.89 \%$ of success.

Analyzing in more detail this last trajectory, which has 191 test points, the RPA has positioned in 109 points $(60.22 \%)$ with five beacons, in 43 points $(22.51 \%)$ with four beacons and in 7 points (3.6\%) with three beacons. On the other hand, the HAPA has positioned in 86 points $(45.03 \%)$ with five beacons, in 71 points $(37.17 \%)$ with four beacons and in 30 points $(15.71 \%)$ with three beacons. The decrease in positions obtained with five beacons by the HAPA is due to the higher sensitivity of this algorithm to cross-talk. As opposed to the RPA, where only two orthogonal codes were used, five codes have to be detected, leading to more incorrect beacon detections. Nevertheless, a significant increase in the availability of $14.64 \%$ is observed, as more positions are obtained with a lower number of beacons, thanks to the additional benefits provided by the HAPA algorithm compared to the RPA. These results also indicate that the HAPA's increased success rate is based on its ability to position in circumstances where the system has to cope with the loss of one or even two beacons. As mentioned, these circumstances occur mainly in areas with low coverage or strongly affected by multipath, as it is the case in this trajectory.

In the above trajectories, the robot always starts from the centre of the positioning area, where it has good coverage, and the algorithm quickly includes correct ToA in the log, which helps to detect multipath in more challenging areas of the trajectory later on. In the following tests, the algorithm's performance is evaluated when starting from low coverage areas, where positioning is more challenging. In these circumstances, the algorithm is not supported by the ToA log from the beginning. Figure $8 \mathrm{~b}$ shows the results of a test in which the robot starts a trajectory at point $(-1.2,1.2)$, describing a clockwise square spiral path that converges to the center of the positioning area. At this path's beginning, there is a gap of $0.4 \mathrm{~m}$ until the system is able to obtain the first position, approximately at point $(-0.8,1.2)$. During this first stretch, the algorithm cannot position the robot because of the low coverage and strong multipath, which the ToA log cannot detect yet, since it needs a position obtained when the five beacons are used. Once the algorithm has managed to obtain positions with all the beacons, it adds the ToA to the log, and its performance stays robust until the end of the trajectory. The positions obtained by the CPA (red crosses in Figure 8b ) are similar in accuracy, but its availability is significantly smaller.

The benefits of the ToA log on positioning in complicated areas is more evident when moving through the environment. An additional test has been conducted, in which the robot starts from the point $(-0.8,1.6)$, a place with low coverage and strong 
multipath where the system has difficulties in positioning. Then the robot make two counterclockwise rounds through a rectangular trajectory until it returns to the starting point. The results obtained can be observed in Figure $8 \mathrm{c}$. In the initial part of the first round (blue circles), some positions are missing due to the absence of entries in the ToA log, so multipath cannot be corrected. Subsequently, when leaving the problematic area, the algorithm starts to position consistently during the rest of the trajectory, adding information to the ToA log. In the second pass (red circles) through the complicated initial zone in the upper-left corner, the presence of red circles indicates that the algorithm has managed to position the robot during this second round. So, even if the system initially might not be able to obtain positions in certain areas due to multipath and low SNR, by moving around in the environment, the user could collect enough information to aid in the positioning later.

\section{Conclusions}

An acoustic positioning system for mobile robots has been developed in this work. This system provides a high accuracy and availability rate in environments with low signal coverage and/or affected by multipath. We developed a High Availability Positioning Algorithm (HAPA) that exploits the information provided by the received signal optimally. This signal comprises a $40 \mathrm{kHz}$ carrier to estimate the Doppler shift, a chirp to determine the Time of Arrival, and a 63-bit Kasami sequence to identify the beacon and validate the correlation peak of its associated chirp. For each of the signals obtained, the algorithm determines the validated set of ToA, corrects their Doppler shift and compensates for multipath if it exists. When there are not enough validated ToA to obtain a position, the algorithm proposes the candidates ToA for the remaining beacons, and it tries to obtain a position by searching for a valid combination of ToA.

The HAPA performance has been evaluated through a set of experimental tests to compare its results with those obtained by the Robust Positioning Algorithm (RPA) developed in [32] and by a Classical Positioning Algorithm (CPA) based only on chirps. A system characterization conducted on a 49-point test grid has shown that the average HAPA's availability in the whole positioning area improves up to $14.77 \%$ compared to the RPA and up to $53.83 \%$ regarding the CPA, increasing these improvements to $29.93 \%$ and $72.83 \%$ respectively, in the peripheral areas. The HAPA also reduces the minimum and maximum mean positioning errors, $1.0 \mathrm{~cm}$ and $4.7 \mathrm{~cm}$ respectively over the RPA, and $1.1 \mathrm{~cm}$ and $0.4 \mathrm{~cm}$ over the CPA. In addition, HAPA achieves position with Signal to Noise Ratio (SNR) as low as $-8 \mathrm{~dB}$, while RPA only achieves positioning with SNR values above $4.5 \mathrm{~dB}$. Besides, a set of tests in motion has been done on various trajectories. The performance improvement offered by the HAPA over the RPA and CPA has also been demonstrated. In particular, on the same 2-meter-side trajectory crossing areas with low coverage and affected by multipath, the HAPA availability is $97.89 \%$, while the RPA drops to $83.25 \%$ and the CPA to $50.19 \%$.

\section{ACKNOWLEDGMENT}

This work has been supported by: the Spanish Government and the European Regional Development Fund (ERDF) through Project MICROCEBUS under Grant RTI2018095168-B-C54, the Regional Government of Extremadura and ERDF-ESF under Project GR18038, and the Research Council of Norway, under project 269614.

\section{REFERENCES}

[1] A. D. Cheok and L. Yue, "A novel light-sensor-based information transmission system for indoor positioning and navigation," IEEE Trans. on Instrumentation and Measurement, vol. 60, no. 1, pp. 290-299, Jan. 2011.

[2] Y. Zhou, C. L. Law, Y. L. Guan, and F. Chin, "Indoor elliptical localization based on asynchronous UWB range measurement," IEEE Trans. on Instrumentation and Measurement, vol. 60, no. 1, pp. 248 257, Jan. 2011.

[3] W. Suski, S. Banerjee, and A. Hoover, "Using a map of measurement noise to improve UWB indoor position tracking," IEEE Trans. on Instrumentation and Measurement, vol. 62, no. 8, pp. 2228-2236, Aug. 2013.

[4] A. Cazzorla, G. De Angelis, A. Moschitta, M. Dionigi, F. Alimenti, and P. Carbone, "A 5.6-GHz UWB position measurement system," IEEE Trans. on Instrumentation and Measurement, vol. 62, no. 3, pp. 675683, Mar. 2013.

[5] G. De Angelis, V. Pasku, A. De Angelis, M. Dionigi, M. Mongiardo, A. Moschitta, and P. Carbone, "An indoor AC magnetic positioning system," IEEE Trans. on Instrumentation and Measurement, vol. 64, no. 5, pp. 1275-1283, May 2015.

[6] B. Kim and S.-H. Kong, "A novel indoor positioning technique using magnetic fingerprint difference," IEEE Trans. on Instrumentation and Measurement, vol. 65, no. 9, pp. 2035-2045, Sept. 2016.

[7] R. Kusche, S. O. Schmidt, and H. Hellbrück, "Indoor positioning via artificial magnetic fields," IEEE Transactions on Instrumentation and Measurement, vol. 70, pp. 1-9, 2021.

[8] C.-H. Huang, L.-H. Lee, C. C. Ho, L.-L. Wu, and Z.-H. Lai, "Realtime RFID indoor positioning system based on Kalman-filter drift removal and Heron-bilateration location estimation," IEEE Trans. on Instrumentation and Measurement, vol. 64, no. 3, pp. 728-739, Mar. 2015.

[9] A. Perttula, H. Leppäkoski, M. Kirkko-Jaakkola, P. Davidson, J. Collin, and J. Takala, "Distributed indoor positioning system with inertial measurements and map matching," IEEE Trans. on Instrumentation and Measurement, vol. 63, no. 11, pp. 2682-2695, Nov. 2014.

[10] J. C. Prieto, A. R. Jiménez, J. Guevara, J. L. Ealo, F. Seco, J. O. Roa, and F. Ramos, "Performance evaluation of 3D-Locus advanced acoustic LPS," IEEE Trans. on Instrumentation and Measurement, vol. 58, no. 8, pp. 2385-2395, Aug. 2009.

[11] R. Zhang, F. Höflinger, and L. Reindl, "TDOA-based localization using interacting multiple model estimator and ultrasonic transmitter/receiver," IEEE Trans. on Instrumentation and Measurement, vol. 62, no. 8, pp. 2205-2214, Aug. 2013.

[12] S. Murano, M. C. Pérez, D. Gualda, F. J. Álvarez, T. Aguilera, and C. De Marziani, "Evaluation of Zadoff-Chu, Kasami and chirp based encoding schemes for acoutic local positioning systems," IEEE Transactions on Instrumentation and Measurements, vol. 69, no. 8, pp. 5356-5368, 2020.

[13] A. Ward, A. Jones, and A. Hopper, "A new location technique for the active office," IEEE Personal Communications, vol. 4, no. 5, pp. 42-47, 1997.

[14] E. Foxlin and M. Harrington, "Constellation: a wide-range wireless motion-tracking system for augmented reality and virtual set applications," in Proceedings of the 25th Annual Conference on Computer Graphics and Interactive Techniques, 1998, pp. 371-378.

[15] N. B. Priyantha, A. Chakraborty, and H. Balakrishnan, "The Cricket location-support system," in Proceedings of the 6th Annual International Conference on Mobile Computing and Networking, 2000, pp. 32-43.

[16] R. Mautz, Indoor Positioning Technologies [Habilitation Thesis]. Institute of Geodesy and Photogrammetry, Department of Civil, Environmental and Geomatic Engineering, ETH Zurich, 2012.

[17] F. J. Álvarez, "Fundamentals of airborne acoustic positioning systems," in Geographical and Fingerprinting Data to Create Systems for Indoor Positioning and Indoor/Outdoor Navigation, J. Conesa, A. Pérez, J. Torres, and R. Montoliu, Eds. Elsevier AP, 2018, ch. 17, pp. 335-352. 
[18] M. Hazas and A. Ward, "A novel broadband ultrasonic location system," in UbiComp 2002: Ubiquitous Computing: 4th International Conference Göteborg, Sweden, September 29 - October 1, 2002 Proceedings, 2002, pp. 264-280.

[19] J. Ureña, A. Hernández, A. Jiménez, J. Villadangos, M. Mazo, J. C. García, J. J. García, F. J. Álvarez, C. De Marziani, M. C. Pérez, J. A. Jiménez, A. R. Jiménez, and F. Seco, "Advanced sensorial system for an acoustic LPS," Microprocessors and Microsystems, vol. 31, no. 6, pp. 393-401, 2007.

[20] D. F. Albuquerque, J. M. Vieira, S. I. Lopes, C. A. Bastos, and P. J. Ferreira, "OFDM pulse design with low PAPR for ultrasonic location and positioning systems," in IEEE International Conference on Indoor Positioning and Indoor Navigation, 2013, pp. 34-35.

[21] F. Seco, J. C. Prieto, A. R. J. Ruiz, and J. Guevara, "Compensation of multiple access interference effects in CDMA-based acoustic positioning systems," IEEE Transactions on Instrumentation and Measurement, vol. 63, no. 10, pp. 2368-2378, 2014.

[22] F. J. Álvarez, T. Aguilera, and R. López-Valcarce, "CDMA-based acoustic local positioning system for portable devices with multipath cancellation," Digital Signal Processing, vol. 62, no. Supplement C, pp. $38-51,2017$.

[23] T. Aguilera, F. J. Álvarez, D. Gualda, J. M. Villadangos, A. Hernández, and J. Ureña, "Multipath compensation algorithm for TDMA-based ultrasonic local positioning systems," IEEE Transactions on Instrumentation and Measurement, vol. 67, no. 5, pp. 984-991, 2018.

[24] T. Aguilera, J. A. Paredes, F. J. Álvarez, J. I. Suárez, and A. Hernández, "Acoustic local positioning system using an iOS device," in International Conference on Indoor Positioning and Indoor Navigation, 2013, pp. 1-8.

[25] D. Gualda, M. C. Pérez-Rubio, J. Ureña, S. Pérez-Bachiller, J. M. Villadangos, A. Hernández, J. J. García, and A. Jiménez, "LOCATEUS: Indoor positioning for mobile devices using encoded ultrasonic signals, inertial sensors and graph-matching," Sensors, vol. 21, no. 6 , 2021. [Online]. Available: https://www.mdpi.com/1424-8220/21/6/1950

[26] M. Benyamin and G. H. Goldman, "Acoustic detection and tracking of a Class I UAS with a small tetrahedral microphone array," Army Research Lab Adelphi MD, Tech. Rep., 2014.

[27] J. Büsset, F. Perrodin, B. Ott, K. Heutschi, T. Rühl, and T. Nussbaumer, "Detection and tracking of drones using advanced acoustic cameras," in Proc. SPIE, vol. 9647, 2015, pp. 9647-9647.

[28] J. A. Paredes, F. J. Álvarez, T. Aguilera, and J. M. Villadangos, "3D indoor positioning of UAVs with spread spectrum ultrasound and timeof-flight cameras," Sensors, vol. 18, no. 1, article no. 89, 2018.

[29] M. Alloulah and M. Hazas, "An efficient CDMA core for indoor acoustic position sensing," in IEEE International Conference on Indoor Positioning and Indoor Navigation, 2010, pp. 1-5.

[30] J. A. Paredes, T. Aguilera, F. J. Álvarez, J. Lozano, and J. Morera, "Analysis of Doppler effect on the pulse compression of different codes emitted by an ultrasonic LPS," Sensors, vol. 11, no. 11, pp. $10765-$ 10784, 2011.

[31] T. Aguilera, F. J. Álvarez, J. A. Paredes, and J. A. Moreno, "Doppler compensation algorithm for chirp-based acoustic local positioning systems," Digital Signal Processing, vol. 100, no. ID 102704, pp. 1-11, 2020.

[32] J. Aparicio, T. Aguilera, and F. J. Álvarez, "Robust airborne ultrasonic positioning of moving targets in weak signal coverage areas," IEEE Sensors Journal, vol. 20, no. 21, pp. 13119-13 130, 2020.

[33] NXP LPC1768, "ARM microcontroller," available online: https://www. nxp.com/docs/en/data-sheet/LPC1769_68_67_66_65_64_63.pdf, last accessed on May 27th, 2021.

[34] Prowave 328ST/R160, "Air ultrasonic ceramic transducers," available online: http://www.prowave.com.tw/english/products/ut/open-type/ 328s160.htm, last accessed on May 27th, 2021.

[35] Bosch GLM 80, "Laser rangefinder," available online: http:// www. bosch - professional.com/sa/en/laser-measure - glm - 80 - 131500 0601072370.html, last accessed on May 27th, 2021.

[36] T. Kasami, "Weight distribution formula for some class of cyclic codes," Coordinate Science Laboratory, Report R-285, 1966.

[37] C. Tseng and C. L. Liu, "Complementary sets of sequences," IEEE Transactions on Information Theory, vol. IT-18, no. 5, pp. 644-652, 1972.

[38] J. A. C. Bingham, "Multicarrier modulation for data transmission: An idea whose time has come," IEEE Communications Magazine, vol. 28, no. 5 , pp. $5-14,1990$

[39] GRAS 40BE 1/4, “Ultrasonic microphone," available online: https : / / www . gras . dk / products / measurement - microphone cartridge / prepolarized - cartridges - 0 - volt / product / 158 - 40be, last accessed on May 27th, 2021.
[40] N. Yoder, "Peakfinder," available online: https://www.mathworks. com/matlabcentral/fileexchange/25500 - peakfinder- x0 -- sel-- thresh -extrema--includeendpoints--interpolate-, last accessed on May 27th, 2021.

[41] Avisoft UltraSoundGate 116H basic, "Recording interface," available online: http://www.avisoft.com/ultrasoundgate/116hb/, last accessed on May 27th, 2021.

[42] Avisoft UltraSoundGate 1/4" , "Mic power module," available online: http://www.avisoft.com/ultrasound-microphones/1-4-mic-powermodule/, last accessed on May 27th, 2021.

[43] Raspbian, "Debian Based Operating System," available online: https: //www.raspbian.org, last accessed on May 13th, 2021.

[44] Aria Library, "Advanced Robotics Interface for Applications," available online: http://wiki.ros.org/Aria, last accessed on May 13th, 2021.

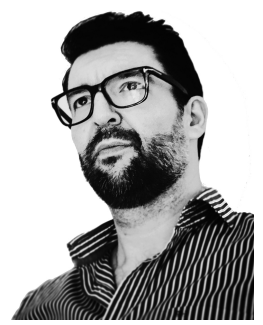

Teodoro Aguilera received his Physics degree in 2009, his M.Sc. in Physics in 2011 and his PhD. in Electronics in 2016, all of them from the University of Extremadura (Spain). Since 2009 he is with the Department of Electrical Engineering, Electronics and Automation at the University of Extremadura where he has worked as an Associate Lecturer and also as Research Fellow of the Sensory Systems Group of this university. Currently his work lies in the design of Acoustic Local Positioning Systems (ALPS) and signal processing.

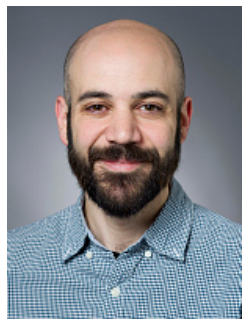

Joaquín Aparicio (M'17) received his Physics degree from the University of Extremadura, Spain, in 2008, and his Ph.D. degree in Electronics from the University of Alcalá, Spain, in 2014. From 2015 to 2018 he was a Postdoctoral Researcher at the Japan Agency for Marine-Earth Science and Technology, Yokosuka, Japan. Since 2018 he is a Postdoctoral Fellow at the Department of Informatics, University of Oslo, Oslo, Norway. His research interests are positioning systems, acoustic propagation, signal processing and communication systems.

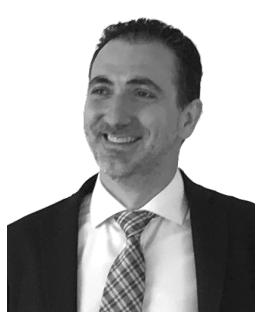

Fernando J. Álvarez (M'07 - SM'17) received his Physics degree from the University of Sevilla, Spain, his Electronic Engineering degree from the University of Extremadura, Spain, and his Ph.D. degree in Electronics from the University of Alcalá, Spain. He also holds a M.Sc. degree in Signal Theory and Communications from the University of Vigo, Spain, and a M.Sc. degree in Telecommunications Engineering from the Universitat Oberta de Catalunya, Spain. Since 2001, he has been with the Department of Electrical Engineering, Electronics and Automation at the University of Extremadura, where he is currently a Full Professor and Head of the Sensory Systems Research Group. In 2008, he joined the Intelligent Sensors Laboratory, Yale University, New Haven, CT, USA, as a Post-Doctoral "José Castillejo" Fellow. His current research interests include local positioning systems, acoustic signal processing, and embedded computing. 